\title{
A 115-bp MethyLight assay for detection of $p 16$ (CDKN2A) methylation as a diagnostic biomarker in human tissues
}

\author{
Jing Zhou', Jie Cao², Zheming Lu', Hongwei Liu² and Dajun Deng ${ }^{1 *}$
}

\begin{abstract}
Background: $p 16$ Methylation is a potential biomarker for prediction of malignant transformation of epithelial dysplasia. A probe-based, quantitative, methylation-specific PCR (MSP) called MethyLight may become an eligible method for detecting this marker clinically. We studied oral mucosa biopsies with epithelial dysplasia from 78 patients enrolled in a published 4-years' followup cohort, in which cancer risk for patients with p16 methylationpositive dysplasia was significantly higher than those without p16 methylation (by 150-bp MSP and bisulfite sequencing; $+133 \sim+283$, transcription starting site, +1$)$. The $p 16$ methylation status in samples $(N=102)$ containing sufficient DNA was analyzed by the 70-bp classic $(+238 \sim+307)$ and 115-bp novel $(+157 \sim+272)$ MethyLight assays, respectively.

Results: p16 Methylation was detectable in 75 samples using the classic MethyLight assay. The methylated-p16 positive rate and proportion of methylated-p16 by the MethyLight in MSP-positive samples were higher than those in MSP-negative samples (positive rate: $37 / 44$ vs. $38 / 58, P=0.035$, two-sided; proportion [median]: 0.78 vs. 0.02 , $P<0.007$ ). Using the published results of MSP as a golden standard, we found sensitivity, specificity, and accuracy for this MethyLight assay to be $70.5 \%, 84.5 \%$, and $55.0 \%$, respectively. Because amplicon of the classic MethyLight procedure only partially overlapped with the MSP amplicon, we further designed a 115-bp novel MethyLight assay in which the amplicon on the sense-strand fully overlapped with the MSP amplicon on the antisense-strand. Using the 115-bp MethyLight assay, we observed methylated-p16 in 26 of 44 MSP-positive samples and 2 of 58 MSPnegative ones $(P=0.000)$. These results were confirmed with clone sequencing. Sensitivity, specificity, and accuracy using the 115-bp MethyLight assay were 59.1\%, 98.3\%, and 57.4\%, respectively. Significant differences in the oral cancer rate were observed during the followup between patients ( $\geq 60$ years) with and without methylated-p16 as detected by the 115-bp MethyLight assay (6/8 vs. 6/22, P =0.034, two-sided).
\end{abstract}

Conclusions: The 115-bp MethyLight assay is a useful and practical assay with very high specificity for the detection of p16 methylation clinically.

\section{Background}

Aberrant methylation of $\mathrm{CpG}$ islands is a very stable modification of genomic DNA that often inactivates gene expression pathologically. Methylation of a target CpG island in even $0.1 \%$ of a cell population obtained from fixed/frozen tissues or body fluids can be detected readily. The high stability and high sensitivity of detection make

\footnotetext{
* Correspondence: dengdajun@bjmu.edu.cn

${ }^{1}$ Key Laboratory of Carcinogenesis and Translational Research (Ministry of Education), Department of Aetiology, Peking University Cancer Hospital \& Institute, Beijing, 100142, China

Full list of author information is available at the end of the article
}

DNA methylation one kind of optimal clinical biomarker for the prediction of potential malignancy progression of precancerous lesions, metastasis/recurrence of cancer, and chemo/radio-therapy sensitivity [1].

It is well recognized that complete methylation of CpG sites within $\mathrm{CpG}$ islands around transcription start sites represents deep-silencing of gene expression established during embryo development and cell differentiation. Well-documented examples include the silencing of tissue-specific genes, gene imprinting, inactivation of parasite DNA and X-chromosome. However, the methylation of $\mathrm{CpG}$ islands in tumor suppressor genes,

\section{Biomed Central}


including $p 16$, is a progressive process encountered during carcinogenesis [2-4]. De novo methylation often occurs post gene silencing at a few seeding CpG sites in initiation and precancerous stages, and ultimately extends to the full $\mathrm{CpG}$ island in advanced cancer. This complicates the development of an assay to detect the methylation status of a target CpG island in which complete methylation is not established. For example, methylation of crucial CpG sites within a CpG island that correlates with clinical outcomes should first be identified, and then a proper detection approach with high specificity for clinical diagnosis should be designed. Unfortunately, such crucial CpG sites are not well characterized for most $\mathrm{CpG}$ islands. This often leads to the dissimilar detection of methylation at different $\mathrm{CpG}$ sites within a target $\mathrm{CpG}$ island between different laboratories. Contradictory results often arise from different kinds of detection assays, or the same assay with different detection sensitivity [5].

Tumor suppressor gene $p 16$ (CDKN2A) controls cell proliferation through the P16-CDK4-RB pathway at the $\mathrm{G} 1 \rightarrow \mathrm{S}$ checkpoint of the cell cycle [6]. Frequent, aberrant methylation of a crucial $\mathrm{CpG}$ island is the main mechanism of inactivation for $p 16$ in the early stages of carcinogenesis [1]. A number of nested case-control studies and followup cohorts consistently showed $p 16$ methylation as a potential biomarker for the early prediction of malignant transformation of epithelial dysplasia, one kind of precancerous lesion in many organs/ tissues including the oral/oesophageal/gastric mucosa [7-13]. Although bisulfite-clone sequencing provides detailed information about the methylation status of each CpG site in the cloning molecules, it is often used as a confirmation assay rather than a regular detection assay because of its low detection sensitivity (> 20\%), labor, and time costs. A number of assays including MSP, MethyLight, Pyrosequencing, and DHPLC are often used to detect $p 16$ methylation in laboratory research [3,7-16]. Among them, MethyLight, based on MSP primers, may become one of the most eligible, convenient, quantitative, and sensitive assays for the clinical detection of $p 16$ methylation primarily because it uses a methylation-specific primer set and real-time, sequence-specific probe validation. In the present study, we evaluated the sensitivity, specificity, and accuracy of a 70-bp classic assay in which the amplicon partially overlapped with the MSP amplicon, and a 115-bp novel MethyLight assay in which the amplicon fully overlapped with the MSP amplicon (Figure 1). The data was collected from 102 oral epithelial dysplasia samples obtained from a followup cohort study, in which malignant transformation of this disease correlated with $p 16$ methylation detected by MSP and was confirmed by clone sequencing [13].

\section{Results and Discussion}

\section{Detection of $p 16$ methylation by a classic $70-b p$} MethyLight assay

An eligible PCR-based molecular assay for diagnosis should meet several essential requirements including high specificity, real-time validation using a sequence-specific probe, positive confirmation with direct sequencing, and refractory to carry-over contamination. Combination of MethyLight using methylation-specific primers with probes containing an anti-contamination system, composed replacing dTTP with dUTP and the addition of a uracil glycosylase UNG in the PCR reaction mixture, may become an ideal method for the clinical detection of methylation in a specific CpG island. In a 4-year followup cohort, we reported that methylated-p16 was a potential biomarker for early prediction of malignant transformation of oral epithelial dysplasia [13]. Among patients of at least 60 years of age, the sensitivity and specificity of methylated-p16 were $77 \%$ and $78 \%$, respectively. Hall et al. reported similar results [14]. Therefore, the using MethyLight as a clinical assay to detect methylated-p16 was feasible.

The 70-bp classic MethyLight for methylated-p16 was evaluated using either genomic DNA of baseline or followup samples $(n=102)$ from patients enrolled in the mentioned cohort $(n=78)$. After genomic DNA was converted to SafeBis templates as described in the methods section, the methylated-p16 was analyzed with the classic MethyLight. Methylated-p16 was detected in 75 of 102 tested samples. The methylated-p16 MethyLightpositive rate and proportion of methylated-p16 in 44 methylated-p16 MSP-positive samples were higher than those in 58 MSP-negative samples, respectively (positive rate: $37 / 44$ vs. $38 / 58, P=0.035$, two-sided; proportion [median]: 0.78 vs. $0.02, P<0.007)$. Using the prognosisrelated MSP-results of methylated- $p 16$ as a golden standard, we found sensitivity, specificity, and accuracy for the classic MethyLight were 70.5\%, 84.5\%, and 55.0\% with a cut-off point of RCN set at 0.073 , respectively (Figure 2A).

\section{Development of a 115-bp novel MethyLight assay}

After conversion of unmethylated cytosine residues to uracil (or thymine in PCR products; $\mathrm{C} \rightarrow \mathrm{U} / \mathrm{T}$ ) residues, a double stranded DNA molecule is transformed into two non-complementary single-stranded DNA molecules $(C \equiv G \rightarrow U / T \neq G)$, as illustrated in Figure 1. Interestingly, all current methylation detection assays for the $p 16$ CpG islands are designed according to the antisense-strand sequence of the $p 16$ exon-1, while none target the sense-strand. The main reasons may include the good performance of first 150/151-bp MSP-m/u for methylated/unmethylated- $p 16$ in cell line and tissue samples, and the very high content $(111 / 175)$ of thymine 


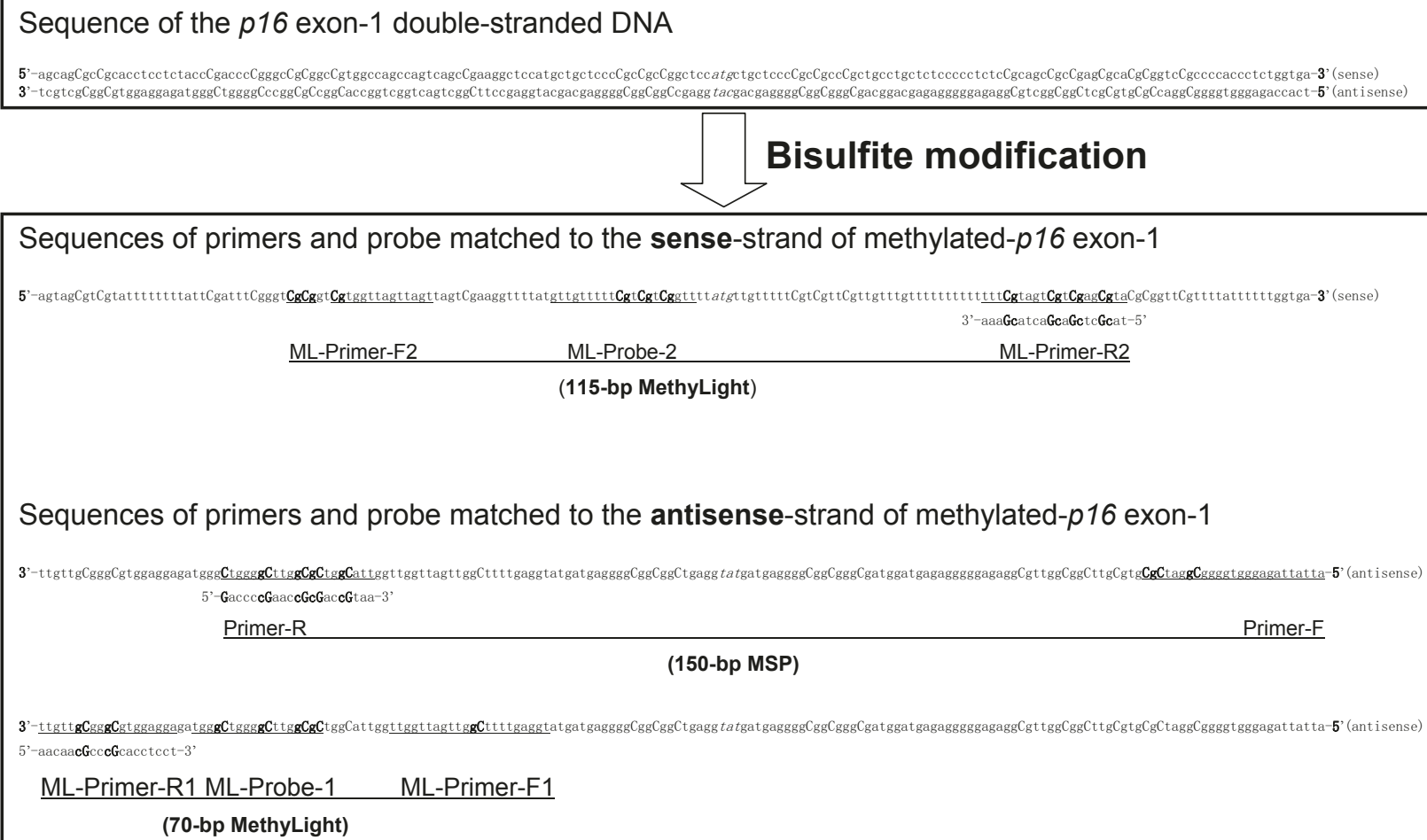

Sequences of primers and probe matched to the antisense-strand of methylated-p16 exon-1

Figure 1 The sequence of the p16 exon-1 before and after bisulfite conversion. Locations of amplicons, primers, and probes used in the 150/151-bp MSP-m/u, 70-bp, and 115-bp MethyLight assays within the sense-strand or antisense-strand were underlined and labelled.

\section{A: 70-bp classic MethyLight}

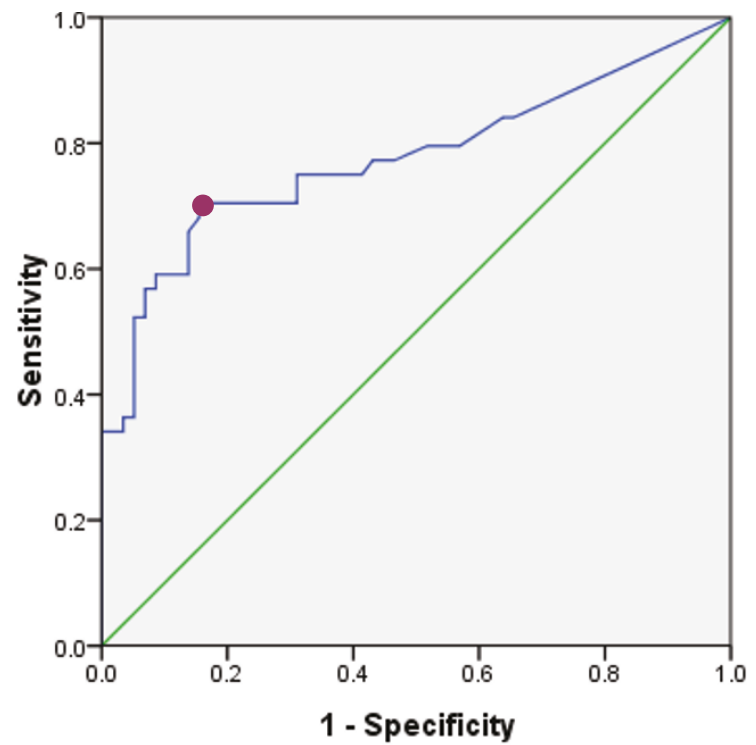

\section{B: 115-bp novel MethyLight}

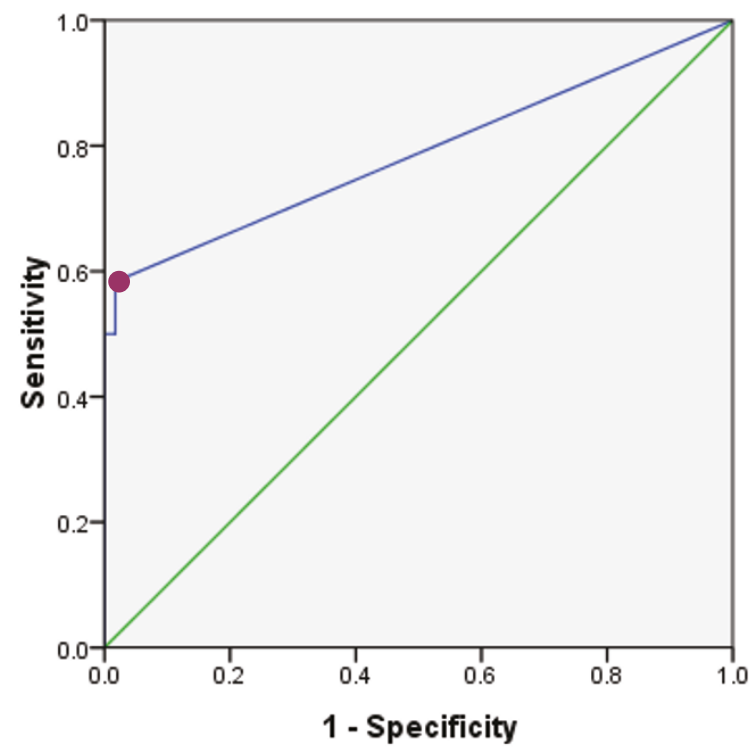

Figure 2 ROC curves of detection of methylated-p16 by two MethyLight assays. The sensitivity and specificity of the MethyLight assay at various points of relative copy number in 102 tested samples were calculated according to the result of the 150-bp MSP. The cut-off points of RCN were marked by the deep-red circlets. A: For the 70-bp classic MethyLight, the area under the curve is 0.776 (95\% Cl: $0.677-0.874)$, P = 0.000 . When the cut-off point of RCN was 0.0725 , the sensitivity and specificity were 0.705 and 0.845 , respectively. B: For the 115 -bp novel MethyLight assay, the area under the curve is 0.787 (95\% Cl: $0.689-0.884)$, $P=0.000$. When the cut-off point of RCN was 0.0002 , the sensitivity and specificity were 0.591 and 0.983 , respectively. 
residues in the unmethylated sense-strand present after bisulfite modification, which makes it difficult to design a proper unmethylation-specific primer set that can be used as control MSP-u in the case that $p-16$ is not methylated (Figure 1). However, in the MethyLight assay, instead of using the template corresponding to unmethylated $p-16$, the COL2A1 gene, without a CpG island, is recommended as an optimal common reference for all tested CpG islands for quantification of modified genomic DNA in the tested samples [17]. Using this strategy, the sense-strand of the methylatedp16 can be used to design a MethyLight assay.

The amplicon $(+238 \sim+307$; transcription starting site, +1 ) of the 70-bp MethyLight partially overlapped with the 150-bp MSP amplicon $(+133 \sim+283)$ (Figure 1). To investigate the feasibility of using the $p 16$ exon- 1 sensestrand for detection of methylated-p16, we designed a 115-bp novel MethyLight assay according to the sensestrand believing it might correlate with the 150-bp MSP better than the 70-bp MethyLight assay. This theory was based on the fact that the 115-bp MethyLight amplicon matched to the 150-bp MSP amplicon better than the 70-bp MethyLight amplicon (Figure 1). Using the novel MethyLight assay, we observed methylated-p16 in 26 of 44 MSP-positive samples and 2 of 58 MSP-negative ones $(P=0.000)$. This result was confirmed by clone sequencing two representative samples (Figure 3). When the RCN cut-off point was set at 0.0002 , the sensitivity, specificity, and accuracy of the 115-bp MethyLight were 59.1\%, $98.3 \%$, and $57.4 \%$, respectively (Figure $2 \mathrm{~B}$ ).

\section{Comparison of two MethyLight assays}

Furthermore, we compared the results of two MethyLight assays and found 23 of the 41 (56.1\%) classic MethyLight positive samples are also 115-bp MethyLight positive; whereas only 4 of $61(6.6 \%)$ classic MethyLight negative samples are 115-bp MethyLight positive $(\mathrm{P}=$ $0.000)$. The detailed overlap information for the results of methylated-p16 in all 102 tested samples was analyzed (Figure 4). Apparently, when the RCN cut-off points were set for the two MethyLight assays, the novel and classic assays had the similar accuracy $(57 \%$ and 55\%). However, the 115-bp MethyLight had a very high specificity, while the classic MethyLight had a higher sensitivity. Most importantly, the sensitivity and specificity of the novel MethyLight assay are consistent regardless of whether the RCN cut-off point was used during the calculation process. This indicates the 115-bp assay could be used as a qualitative assay for clinical detection of methylated-p16.

We further analyzed the clinical outcome of methylated-p16 as detected by two MethyLight assays. Among

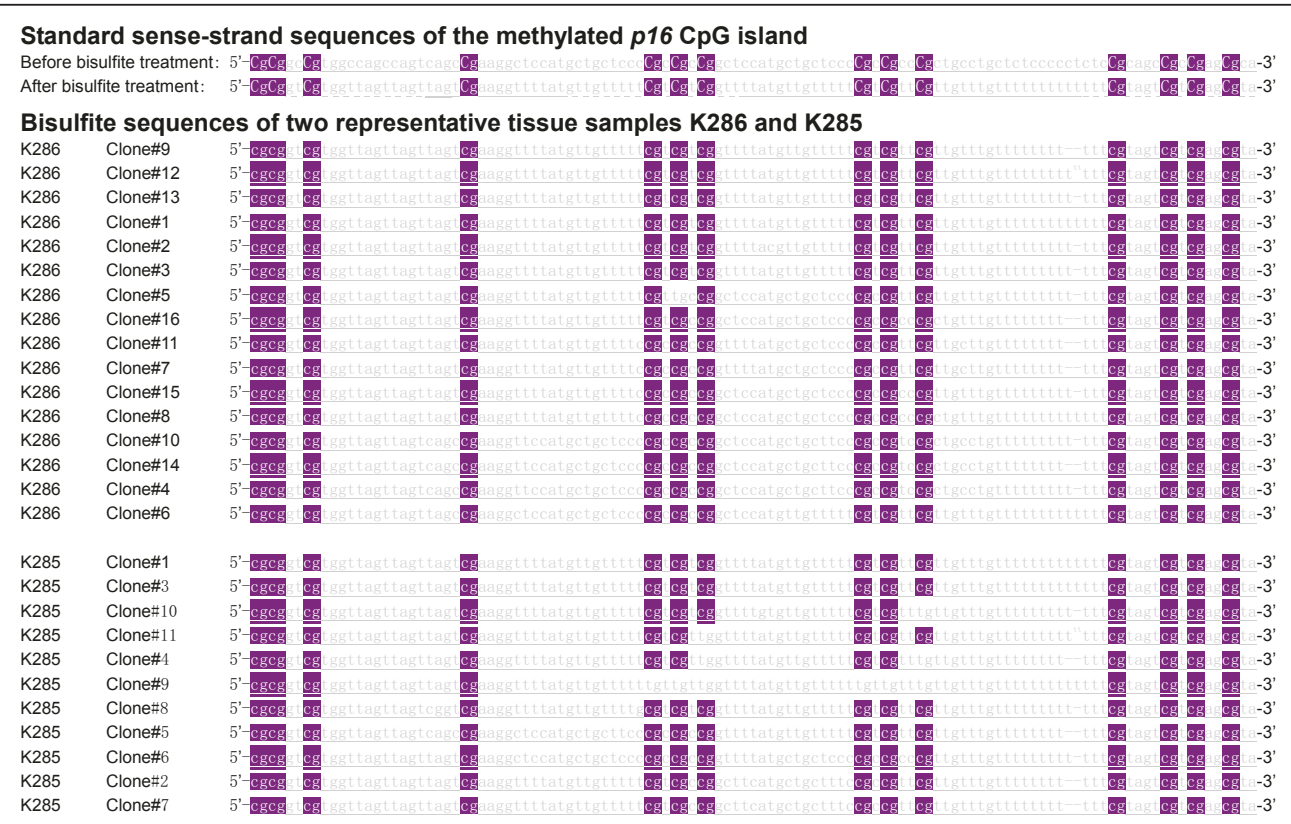

Figure 3 Results of clone sequencing of the 115-bp MethyLight PCR products. The sense-strand sequences of the methylated p16 CpG island with and without bisulfite treatment were listed. The bisulfite-treated template of two 115-bp MethyLight-positive samples was amplified with the same primer set. The PCR products of these two representative samples were clone-sequenced, respectively. 99.6\% (223/224) and 93.5\% (144/154) cytosines at CpG sites within total 16 and 11 clones (14 CpG sites/clone) from the sample K286 and K285 were maintained and 77.8\% (548/704) and 90.9\% (440/484) cytosines at non-CpG sites within these clones (44 non-CpG sites/clone) were converted to thymines, respectively. These results indicate that these clones are fully methylated at all $\mathrm{CpG}$ sites. 


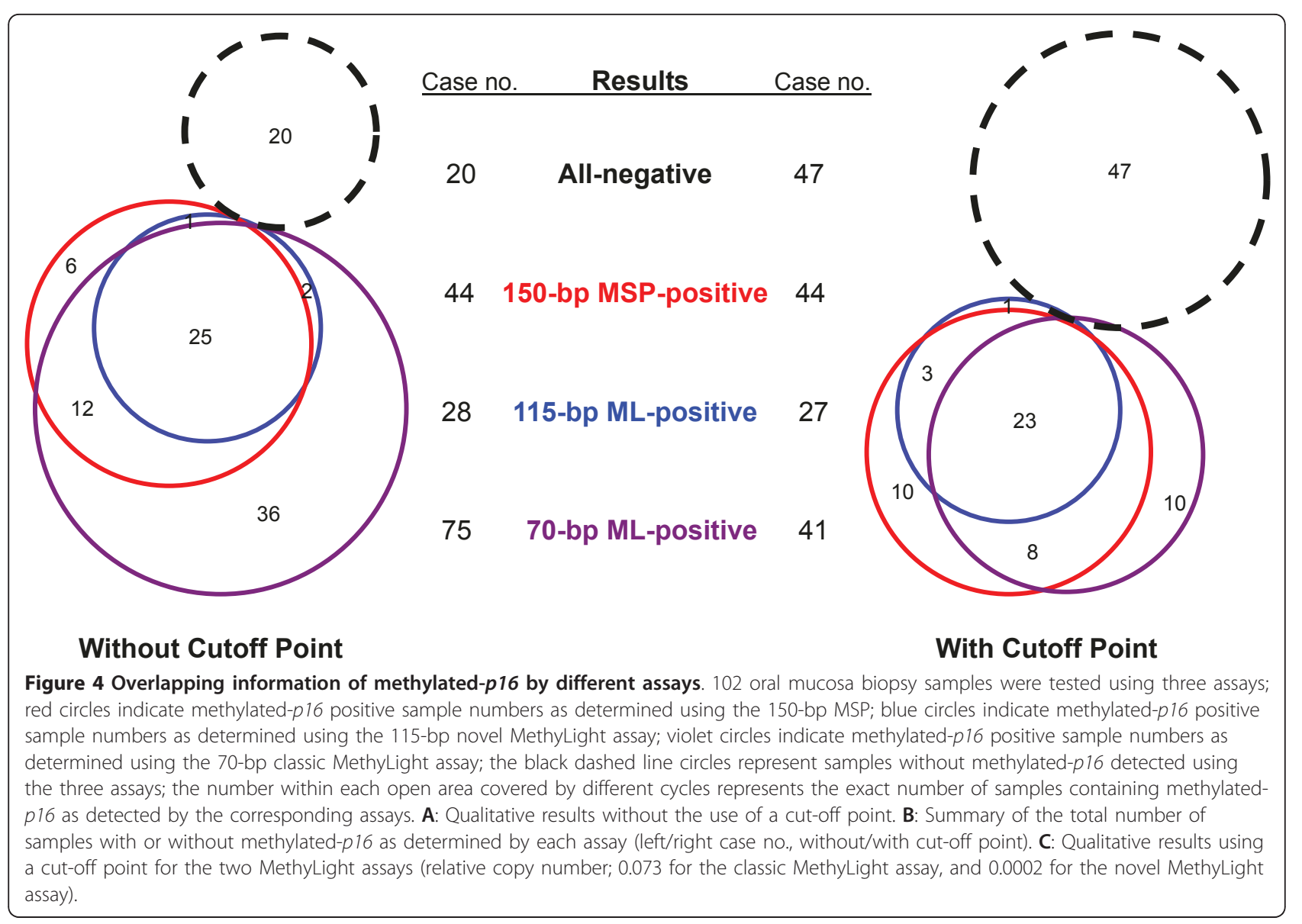

30 patients of at least 60 years of age, methylated-p16 was detected in 8 baseline samples by the 115-bp MethyLight assay (with or without the cut-off value). During the followup period, oral cancer developed in 6 of 8 methylated-p16 positive patients (75.0\%), but only 6 of 22 patients (27.3\%) without methylated-p16 developed oral cancer [odd ratio 8.00 (95\% CI, 0.98 80.93; $\mathrm{P}=0.034$, two-sided). Among 34 patients analyzed using the classic MethyLight assay (with cut-off value $0.073)$, the odds ratio of methylated-p16 was $3.64(6 / 10$ vs. 7/24; 95\% CI, 0.62 21.91; $\mathrm{P}=0.130$ ). These results suggest that the 115-bp MethyLight assay might be better suited to detect the methylated-p16 biomarker than the classic MethyLight assay.

\section{Conclusions}

The 115-bp MethyLight assay maybe a practical assay for the detection of methylated-p16 biomarker for clinical diagnosis.

\section{Methods}

\section{Patients and oral biopsies}

102 genomic DNA samples (> $500 \mathrm{ng}$ ) were extracted from paraffin-embedded oral mucosa biopsies containing mild or moderate dysplasia lesions from 78 patients enrolled in a 4-year follow-up cohort (NCT00835341, available at http://ClinicalTrials.gov) $[7,13]$. Briefly, the fixed tissue block was cut into $10 \mu \mathrm{m}$ slides, treated with xylene to remove the paraffin, rehydrated with graded ethanol, mixed with lysis buffer containing 100 $\mu \mathrm{g}$ proteinase $\mathrm{K}$, digested at $56^{\circ} \mathrm{C}$ overnight, and incubated $10 \mathrm{~min}$ at $95^{\circ} \mathrm{C}$ to stop the digestion [18]. DNA present in the digestion solution was precipitated with ethanol and dissolved in $50 \mu \mathrm{l}$ TE buffer. DNA concentration was determined spectrophotometrically with diphenylamine as described [19]. The average recovery rate of genomic DNA was $77.6 \%$. 61 samples were baseline biopsies and the remaining 41 samples were taken during the followup periods. Methylation status of the antisense-strand of exon- 1 within the $p 16$ CpG island was determined using a 150-bp MSP assay in which DHPLC was used as the detector; the results were further confirmed through clone sequencing (Figure 1). Methylated- $p 16$ was detected in 44 of these samples. The study was approved by the Institutional Review Boards of Peking University School of Stomatology and School of Oncology, and all patients gave written informed consent. 
Preparation of SafeBis DNA by bisulfite treatment

Genomic DNA samples $(2 \mu \mathrm{g})$ were treated with bisulfite for $16 \mathrm{hrs}$ at $50^{\circ} \mathrm{C}$ without desulfonation as described [20], purified with the Wizard DNA Clean-Up System Kit (Promega, Madison, WI), dissolved in $40 \mu \mathrm{l}$ TE preheated to $80^{\circ} \mathrm{C}$, and stored in three aliquots at $-20^{\circ} \mathrm{C}$ before use. The unmethylated cytosine residues in the DNA were converted to uracil (thymine in PCR products) and the methylated cytosine residues remained intact after this treatment.

\section{Detection of $p 16$ methylation by the 70 -bp classic MethyLight assay}

Methylation of CpG sites across the MSP Primer-R region in the antisense-strand of the $p 16$ exon- 1 was analyzed by the classic MethyLight assay using modified primers [15]. Briefly, the ML-Primer-F1 (5'-tggag ttttC ggttg attgg tt-3'), ML-Primer-R1 (5'-aacaa cGccc Gcacc tcct$3^{\prime}$ ), and a methylated-p16-specific ML-Probe-1 (6FAM5'acc $\mathbf{C g}$ accc $\mathbf{C}$ gaacC $\mathbf{g C g}$-3'TAMRA, TaqMan) were used to detect the 70-bp methylated $p 16$ templates in the SafeBis DNA (Figure 1). The reference gene COL2A1 was also amplified with a forward primer (5'-tctaa caatt ataaa ctcca accac caa-3'), a reverse primer (5'-gggaa gatgg gatag aaggg aatat-3'), and a COL2A1-specific probe (6FAM5'ccttc attct aaccc aatac ctatc ccacc tctaa a-3'BHQ1) [17]. A uracil DNA glycosylase (UNG) carry-over prevention system was employed in the MethyLight assay [18]. The $20 \mu \mathrm{l}$ MethyLight reaction mixture contained $2 \mu \mathrm{l}$ $10 \times$ PCR buffer (Qiagen, Germany), 0.5 units of HotStar Taq DNA polymerase (Qiagen), $200 \mu \mathrm{mol} / \mathrm{L}$ dATP, 200 $\mu \mathrm{mol} / \mathrm{L} \mathrm{dCTP}, 200 \mu \mathrm{mol} / \mathrm{L} \mathrm{dGTP}, 800 \mu \mathrm{mol} / \mathrm{L}$ dUTP (Promaga), $5 \mathrm{mmol} / \mathrm{L} \mathrm{MgCl}_{2}, 75 \mathrm{nmol} / \mathrm{L}$ of each primer (TaKaRa, Beijing), $75 \mathrm{nmol} / \mathrm{L}$ probe (TaKaRa), $2 \mu \mathrm{l}$ $10 \times$ UNG Buffer (NEB), 0.4 units UNG (NEB), and $10 \mathrm{ng}$ template. An ABI7500 thermal cycler was used to conduct the $\mathrm{PCR}$ reactions using the following thermal conditions: $37^{\circ} \mathrm{C}$ for $10 \mathrm{~min} \rightarrow 95^{\circ} \mathrm{C}$ for $30 \mathrm{~min} \rightarrow\left(95^{\circ} \mathrm{C}\right.$ for $15 \mathrm{sec} \rightarrow 62^{\circ} \mathrm{C}$ for $\left.1 \mathrm{~min}\right) \times 45$ cycles. The fluorescence value was detected at $62^{\circ} \mathrm{C}$. Duplicate tubes were used for each sample, and the average $\mathrm{Ct}$ value was used in the calculations. Relative copy number (RCN) of methylated- $p 16$ was calculated according to the formula $\left[2^{-\Delta \mathrm{Ct}},\left(\Delta \mathrm{Ct}=\mathrm{Ct}_{\text {methylated-p16}}-\mathrm{Ct}_{\mathrm{COL2A1}}\right)\right]$. RKO and MGC803 xenografts from nude mice were also used as methylated-p16 positive and negative controls in each experiment, respectively [13]. The calculated RCN of methylated-p16 in each sample was standardized according to the RCN of RKO positive control.

\section{Detection of $p 16$ methylation by the 115-bp MethyLight assay}

The ML-Primer-F2 (5'-CgCgg tCgtg gttag ttagt-3'), ML-Primer-R2 (5'-tacGc tcGac Gacta Cgaaa-3'), and
ML-Probe-2 (5'-6FAM-gttgt ttttC gtCgt Cggtt-TAMRA3 ') were used to detect the 115-bp methylated fragment of the sense-strand of $p 16$ exon-1, which completely overlapped the sense-strand sequence corresponding to the 150-bp MSP amplicon within the antisense-strand (Figure 1). Other conditions were the same as the classic MethyLight assay.

\section{Clone sequencing of the 115-bp MethyLight PCR products of methylated- $p 16$}

The SafeBis template from two representative samples of the 115-bp MethyLight-positive samples was amplified with the same primer set used in the 115-bp MethyLight assay (without the ML-Probe-2), and then clone-sequenced as described [3].

\section{Statistical methods}

A ROC curve of the results for each MethyLight assay was calculated. Results of methylated-p16 in these tested samples, determined using the 150-bp MSP-m (and by151-bp MSP-u in the MSP-m negative cases), were used as the golden standard in the calculation of sensitivity and specificity for the two MethyLight assays (Figure 1). These results showed a strong correlation with the malignant transformation of these lesions in the 4-year followup cohort study [13]. The accuracy was calculated according to the formula [Sensitivity+Specificity-1]. The Chi-square test and Student's t-test were used to test the significance of qualitative and quantitative data between different groups. All tests were two-sided.

\section{Acknowledgements}

This work is supported by Capital Program for Development of Health Science (Grant \#434), Beijing Science and Technology Commission (Grant \#Z090507017709016), Key Technologies Research \& Development Programs (863 Grants \#2006AA020902 and 2006AA02A402). We thank Dr. Zhaojun Liu for preparation of ROC curve charts. We also thank Professor Huidong Shi and Mr. James Wilson (Augusta, Georgia) for language editing.

\section{Author details}

${ }^{1}$ Key Laboratory of Carcinogenesis and Translational Research (Ministry of Education), Department of Aetiology, Peking University Cancer Hospital \& Institute, Beijing, 100142, China. ${ }^{2}$ Department of Oral Medicine, Peking University School of Stomatology, Beijing 100081, China.

\section{Authors' contributions}

JZ carried out the molecular epigenetic assays. ZL carried out the clone sequencing. JC and HL collected the tested samples. DD conceived the study and drafted the manuscript. All authors read and approved the final manuscript.

Received: 2 February 2011 Accepted: 13 May 2011

Published: 13 May 2011

\section{References}

1. Deng D, Liu Z, Du Y: Epigenetic alterations as cancer diagnostic, prognostic, and predictive biomarkers. Adv Genet 2010, 71:125-176.

2. Wong DJ, Foster SA, Galloway DA, Reid BJ: Progressive region-specific de novo methylation of the $\mathrm{p} 16 \mathrm{CpG}$ island in primary human mammary 
epithelial cell strains during escape from $\mathrm{M}(0)$ growth arrest. Mol Cell Biol 1999, 19:5642-5651.

3. Luo DY, Zhang BZ, Lv LB, Xiang SY, Liu YH, Ji JF, Deng DJ: Methylation of CpG islands of p16 associated with progression of primary gastric carcinomas. Laboratory Investigation 2006, 86:591-598.

4. Hinshelwood RA, Melki JR, Huschtscha LI, Paul C, Song JZ, Stirzaker C, Reddel RR, Clark SJ: Aberrant de novo methylation of the p16INK4A CpG island is initiated post gene silencing in association with chromatin remodelling and mimics nucleosome positioning. Hum Mol Genet 2009, 18:3098-3109.

5. Capel E, Fléjou JF, Hamelin R: Assessment of MLH1 promoter methylation in relation to gene expression requires specific analysis. Oncogene 2007, 26:7596-7600.

6. Serrano M, Hannon G, Beach D: A new regulatory motif in cell-cycle control causing specific inhibition of cyclin D/CDK4. Nature 1993, 366:704-707.

7. Sun Y, Deng DJ, You WC, Bai H, Zhang L, Zhou J, Shen L, Ma JL, Xie YQ, $\mathrm{Li} \mathrm{JY}$ : Methylation of p16 CpG islands associated with malignant transformation of gastric dysplasia in a population-based study. Clinical Cancer Research 2004, 10:5087-5093.

8. Belinsky SA, Liechty KC, Gentry FD, Wolf HJ, Rogers J, Vu K, Haney J, Kenned TC, Hirsch FR, Miller Y, Franklin WA, Herman JG, Baylin SB, Bunn PA, Byers T: Promoter hypermethylation of multiple genes in sputum precedes lung cancer incidence in a high-risk cohort. Cancer Research 2006, 66:3338-3344.

9. Schulmann K, Sterian A, Berki A, Yin J, Sato F, Xu Y, Olaru A, Wang S, Mori Y, Deacu E, Hamilton J, Kan T, Krasna MJ, Beer DG, Pepe MS, Abraham JM, Feng Z, Schmiegel W, Greenwald BD, Meltzer SJ: Inactivation of p16, RUNX3, and HPP1 occurs early in Barrett's-associated neoplastic progression and predicts progression risk. Oncogene 2005, 24:4138-4148.

10. Wang JS, Guo M, Montgomery EA, Thompson RE, Cosby H, Hicks L, Wang S, Herman JG, Canto MI: DNA promoter hypermethylation of p16 and APC predicts neoplastic progression in Barrett's esophagus. Am J Gastroenterol 2009, 104:2153-2160

11. Jin Z, Cheng Y, Gu W, Zheng Y, Sato F, Mori Y, Olaru A, Paun B, Yang J, Kan T, Ito T, Hamilton JP, Selaru FM, Agarwal R, David S, Abraham JM, Wolfsen HC, Wallace MB, Shaheen NJ, Washington K, Wang J, Canto MI, Bhattacharyya A, Nelson MA, Wagner PD, Romero Y, Wang KK, Feng Z, Sampliner RE, Meltzer SJ: A multicenter, double-blinded validation study of methylation biomarkers for progression prediction in Barrett's esophagus. Cancer Res 2009, 69:4112-4115.

12. Hall G, Shaw R, Field E, Rogers S, Sutton D, Woolgar J, Lowe D, Liloglou T, Field J, Risk J: p16 Promoter methylation is a potential predictor of malignant transformation in oral epithelial dysplasia. Cancer Epidemiol Biomarkers Prev 2008, 17:2174-2179.

13. Cao J, Zhou J, Gao Y, Gu LK, Meng HX, Liu HW, Deng DJ: Methylation of p16 CpG Island Associated with Malignant Progression of Oral Epithelial Dysplasia: A Prospective Cohort Study. Clinical Cancer Research 2009, 15:5178-5183.

14. Herman JG, Graff JR, Myohanen S, Nelkin BD, Baylin SB: Methylationspecific PCR: A novel PCR assay for methylation status of CpG islands. Proceedings of the National Academy of Sciences of the United States of America 1996, 93:9821-9826.

15. Eads CA, Lord RV, Kurumboor SK, Wickramasinghe K, Skinner ML, Long TI, Peters JH, DeMeester TR, Danenberg KD, Danenberg PV, Laird PW, Skinner KA: Fields of aberrant CpG island hypermethylation in Barrett's esophagus and associated adenocarcinoma. Cancer Res 2000, 60:5021-5026.

16. Shaw RJ, Akufo-Tetteh EK, Risk JM, Field JK, Liloglou T: Methylation enrichment pyrosequencing: combining the specificity of MSP with validation by pyrosequencing. Nucleic Acids Res 2006, 34:e78.

17. Widschwendter M, Siegmund KD, Müller HM, Fiegl H, Marth C, MüllerHolzner E, Jones PA, Laird PW: Association of breast cancer DNA methylation profiles with hormone receptor status and response to tamoxifen. Cancer Res 2004, 64:3807-3813.

18. Dieffenbach CW, Dveksler GS: PCR Primer: A Laboratory Manual. New York: Cold Spring Harbor Laboratory Pressi, 11995.

19. Furihata C, Yamawaki Y, Jin SS, Moriya H, Kodama K, Matsushima T, Ishikawa T, Takayama S, Nakadate M: Induction of unscheduled DNA synthesis in rat stomach mucosa by glandular stomach carcinogens. J Natl Cancer Inst 1984, 72:1327-1334.
20. Tetzner R, Dietrich $D$, Distler J: Control of carry-over contamination for PCR-based DNA methylation quantification using bisulfite treated DNA. Nucleic Acids Res 2007, 35:e4.

\section{Pre-publication history}

The pre-publication history for this paper can be accessed here: http://www.biomedcentral.com/1471-2350/12/67/prepub

doi:10.1186/1471-2350-12-67

Cite this article as: Zhou et al:: A 115-bp MethyLight assay for detection of $p 16$ (CDKN2A) methylation as a diagnostic biomarker in human tissues. BMC Medical Genetics 2011 12:67.

\section{Submit your next manuscript to BioMed Central and take full advantage of:}

- Convenient online submission

- Thorough peer review

- No space constraints or color figure charges

- Immediate publication on acceptance

- Inclusion in PubMed, CAS, Scopus and Google Scholar

- Research which is freely available for redistribution

Submit your manuscript at www.biomedcentral.com/submit
Biomed Central 